\title{
ORIGINAL
}

ARTICLES

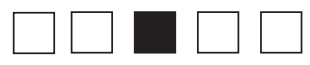

\section{The Impact of Physician Demographic Characteristics on Perceptions of Their Attire}

Paula Varnado-Sullivan, PhD; Michele Larzelere, PhD; Kaly Solek, MA; Shalinder Gupta, MD; Jackson E. Hatfield, MD; Mohammad H. Cheema, MD; Matthew Delfino, MD, MBA

\begin{abstract}
BACKGROUND AND OBJECTIVES: Few studies address the impact of physician attire on ratings of personality characteristics in the presence of varied physician demographic characteristics (gender, racial/ethnic background). Even fewer have examined the boundaries of acceptable physician attire, given recent loosening of societal standards of dress.
\end{abstract}

METHODS: Using an online survey methodology, adult participants ( $\mathrm{N}=505$; 45\% medical professionals) were recruited. Participants rated target photos depicting a male and female individual from three ethnic/racial categories each dressed in business casual (with and without a white coat) or in professional attire (with and without a white coat) on a number of personality characteristics. General willingness to have physicians wear certain apparel items was also queried, as was the importance/acceptability of specific clothing items and appearance choices. Responses were analyzed by gender, age, ethnicity, and profession of respondent.

RESULTS: Both business casual and professional attire were rated highly. A name tag had the highest ratings for importance of wear. The results for wearing a white coat were not as consistent as earlier studies as physicians were perceived as warmer and kinder when not wearing a lab coat, particularly with professional attire. However, female Caucasian physicians were rated most positively when wearing a lab coat. Consistent with previous studies, attire that was too casual (jeans, t-shirts) was rated negatively.

CONCLUSIONS: The current study supports the notion that rules of attire are changing, even in the physician's office. Name tags were perceived to be crucial in medical settings, and casual clothing should be avoided. Despite often being considered a defining component of a physician's "uniform," the white lab coat may not be a universal positive and perhaps even a negative for some physicians.

(Fam Med. 2019;51(9):737-41.) doi: 10.22454/FamMed.2019.650493

Published Online First August 26, 2019

$\Delta$ ppearance plays a significant role in social interactions. For example, positive personality characteristics are often attributed to people rated as attractive, while negative attributes are assigned to individuals perceived to

unattractive. ${ }^{1-2}$ Attire telegraph cultural markers such as status and the first markers of professionalism that patients experience. ${ }^{4-5}$ Changes in societal dress standards warrant investigation of patients' current preferences for physician attire. ${ }^{6}$ Historically, patients-particularly older ones-preferred physicians wear professional clothing (collared shirt/tie/ slacks, blouse/skirt or slacks) with a white coat and stethoscope. ${ }^{7-11}$ African-American patients placed greater importance on the appearance of their physician than did Caucasian respondents. ${ }^{8}$ Prior research, however, did not consider how physicianperceived attractiveness or ethnicity might influence results.

Only a few studies of physician attire have considered changing provider demographics. For example, judgements of non-Caucasian and female physicians may be more heavily influenced by clothing choices. ${ }^{8}$ Female professionals may face greater challenges given a wider variability of clothing options and rapidly changing trends. For example, having bare legs with dresses/skirts has not been examined as previous studies specified "dress with nylons." addition, female physicians may be more easily mistaken for other members of the clinical care team when wearing certain clothing items. ${ }^{12}$ Additional research is needed to examine this potential misperception. Physician opinion of colleagues' dress is also important. Even if more profession. ${ }^{3}$ Physician attire is one of
From the Department of Psychology, Southeastern Louisiana University, Hammond, LA (Dr Varnado-Sullivan and Ms Solek), and Louisiana State University Family Medicine Residency, Kenner, LA (Drs Larzelere, Gupta, Hatfield, Cheema, and Delfino). 
casual dress was preferred by patients, standing among one's peers may be diminished by casual dress. Prior research has suggested that physicians favor traditional appearance among their colleagues ${ }^{13}$ with older physicians holding stronger traditionalist views than younger physicians. ${ }^{11}$ However, research to date has not examined physician or patient attitudes about nontraditional forms of physical self-expression (eg, tattoos, that are becoming more common).

Previous studies of physician attire have not controlled for physician appearance. Further, these studies did not consider the changing demographics of the medical profession. Therefore, the purpose of this study was to evaluate perceptions of physician dress by physician gender and race, while accounting for perceived attractiveness. How these characteristics impacted perceived role and comfort with the physician were examined, as were preferred clothing type for physicians and other factors such as wearing a name tag. Based upon prior research, ${ }^{8}$ it was hypothesized that non-Caucasian and female clinicians would be judged more negatively when dressed casually. It was also hypothesized that medical personnel would prefer more traditional physician attire and a more standard physical appearance. It was expected that age would impact ratings for attire and appearance in that both older medical professionals and patients would prefer a more traditional appearance for physicians.

\section{Methods}

An online survey methodology was used and all responses were anonymous. Subjects were recruited through flyers in a primary care outpatient clinic in a southern city, as extra credit opportunities at a southern 4-year college, and via appeals on listservs and emails to garner participation by physicians and others employed in medical settings. Those participants solicited from online sources resided throughout the United States. Participants under
18 years old or not fluent in English were excluded. Insitutional review board approval for the study was obtained from all participating institutions, and participants provided informed consent prior to starting the online survey.

Nonphysicians of similar ages in six demographic categories (male and female in three ethnic/racial categories: Caucasian, African American, Indian American) were photographed in four modes of attire. Nonphysicians were used to ensure participants would not know the pictured physician or target. Attire was business casual (pullover shirt, pants), business casual with a lab coat, professional (button down shirt, pants), and professional with a lab coat. Neutral facial expressions were held. Those photographed were chosen to represent an average appearance that was determined through pilot testing. Finally, participants were asked if they knew the person in the photograph, and yes responses were excluded from analyses.

Participants were randomly assigned to view one of the photographs. After viewing the image, they rated the targets on 15 attributes (eg, kind, competent, professional, intelligent) using a 7-point Likert scale, as well as for perceived attractiveness. Participants were also asked to indicate the assumed health care role of the target (eg, physician, nurse, receptionist, etc) and their comfort discussing physical, emotional, and sexual health with the target. Participants rated the acceptability of a variety of clothing items for physicians across settings and chose their preferred manner of dress for their outpatient physician. Finally, the importance of certain appearance factors (eg, wearing a tie, wearing a name tag, visible piercings, visible tattoos) in their judgment of a physician was assessed.

\section{Results}

Demographics

There were 505 participants $(64 \%$ female, $27 \%$ male, $9 \%$ not specified) with a mean age of 30.03 years $(\mathrm{SD}=13.09$; range 18-78 years). Forty-five percent worked in the medical field (physician 9\%, resident physician $11 \%$, medical student $2 \%$, nurse $5 \%$, allied health $3 \%$, other $15 \%)$. Caucasian/European participants comprised $63 \%$ of the sample. The remainder of the sample self-identified as African American (12\%), Asian/Southeast Asian/Pacific Islander (5\%), Hispanic/Latino (4\%), Indian Subcontinent (3\%), American Indian/Alaskan Native (1\%), Middle Eastern $(1 \%)$ and African $(<1 \%)$. The remainder did not specify or chose "other."

\section{Data Analysis}

Data were analyzed using SPSS 22. Multivariate analyses were used to examine hypotheses related to physician attire. An initial analysis indicated that attractiveness ratings for the images were found to differ significantly, with the female photos, particularly the Caucasian female, being rated more attractive than the males $(F[5,496]=28.25 ; P<.01$ [Table 1]). Therefore, multivariate analyses of covariance (MANCOVA) were conducted with attractiveness entered as the covariate for the primary hypotheses and follow-up analyses by subgroups (medical professionals, age, physicians only). All post-hoc analyses were conducted by examining the Bonferroni-corrected pairwise comparisons of estimated marginal means.

\section{Analyses of Physician Attire}

A principal components factor analysis with Varimax rotation was conducted on the 15 attributes used in rating the target. Two factors were indicated that accounted for $78 \%$ of the variance. Professionalism had an eigenvalue of 10.60 , and included the characteristics professional (.79), intelligent (.79), driven (.80), disciplined (.84), self-confident (.67), competent (.71), capable (.78), and reliable (.72). A composite scale score was created and had a Cronbach $\alpha$ of .95. The scale score could range from 7-56 with higher scores 
indicating more professionalism. The second factor, humanism, had an eigenvalue of 1.09 and included the items honest (.63), trustworthy (.71), likable (.81), friendly (.86), helpful (.71), warm (.88), and kind (.84). For this factor, Cronbach $\alpha$ was .96 and the composite score could range from $7-49$, with higher scores indicating greater levels of humanism. The two factors were significantly correlated $(r=.83 ; P<.01)$. A 4 (outfit type) x 6 (target) MANCOVA with humanism and professionalism as the dependent variables was conducted. It indicated significant main effects for target $(F[10,890]=19.58, P<.01$; outfit type $F[6,890]=4.79, P<.01)$, and the covariate, attractiveness $(F[2$, $444]=68.68 ; P<.01)$, as well as the interaction for target by outfit ( $\mathrm{F}[30$, $890]=3.15, P<.01)$.

For humanism, significant main effects were found for target $(F[5$, $445]=37.40 ; P<.01)$ and outfit type $(F[3,445]=6.30 ; P<.01$ [Tables 1 and 2]). Post-hoc analysis indicated that the Caucasian female target was rated significantly higher than all targets except the African American male. There were no significant differences between the humanism ratings of the African American male, the African American female, and the Caucasian male, however, the African American male had a significantly higher humanism rating than the Indian male $(P<.01)$. All targets scored significantly higher than the Indian female for humanism $(P<.01)$. For outfit type, business casual $(P<.01)$ and professional $(P<.05)$ attire garnered higher scores than professional attire with a lab coat for humanism. When subgroup analyses were performed, business casual attire was rated higher on humanism amongst Caucasian participants $(F[3,327]=3.22 ; P<.05)$, in older participants $(F[3,237]=4.82$; $P<.01$ ), and by those working in the medical field $(F[3,206]=4.34 ; P<.01)$. When physicians were analyzed separately, there was no significant difference in ratings of humanism by type of clothing among younger physicians ( $\leq 35$ years), however, there was a significant ratings difference in older ( $>35$ years) physicians $(F[3$, $34]=4.05 ; P<.05)$. In this subsample, business casual-attired providers were rated higher than both professional with a lab coat and business casual with a lab coat-attired providers on humanism $(P<.05)$.

For professionalism, there was no significant main effect for outfit type, but there was for target, $(F$ $[5,445]=17.00 ; P<.01)$. The nonsignificant finding for outfit type was replicated in subgroup analyses by participant race (Caucasian or minority), age ( $<24$ years or $\geq 24$ years, and employment in the medical field. When physicians were analyzed separately, there was a significant difference for older physicians $(>35$ years, $F[3,34]=3.77 ; P<.05)$. Posthoc comparisons indicated professionally attired providers were rated significantly higher on professionalism than business casual with lab coat $(P<.05)$. Post-hoc analysis for target found that the African American male was rated significantly higher for professionalism than all other targets except the Caucasian female and Indian male. The Caucasian female was also rated higher than the African American female. The Indian female was significantly

Table 1: Attribute Ratings by Target Individual

\begin{tabular}{|l|c|c|c|}
\hline \multicolumn{1}{|c|}{ Target Individual } & $\begin{array}{c}\text { Attractiveness } \\
\text { (Mean, SD) }\end{array}$ & $\begin{array}{c}\text { Humanism } \\
\text { (Mean, SE) }\end{array}$ & $\begin{array}{c}\text { Professionalism } \\
\text { (Mean, SE) }\end{array}$ \\
\hline African American female & $4.50(1.50)$ & $26.47(.74)$ & $38.69(1.21)$ \\
\hline African American male & $3.51(1.48)$ & $28.23(.72)$ & $44.44(1.17)$ \\
\hline Caucasian female & $4.69(1.31)$ & $30.24(.62)$ & $43.70(1.01)$ \\
\hline Caucasian male & $2.81(1.24)$ & $26.18(.66)$ & $38.14(1.08)$ \\
\hline Indian female & $4.27(1.38)$ & $18.91(.64)$ & $32.46(1.05)$ \\
\hline Indian male & $3.07(1.49)$ & $24.77(.68)$ & $39.82(1.11)$ \\
\hline
\end{tabular}

Higher scores indicate higher levels of the attributes.

Table 2: Means and Standard Error for Attribute Ratings by Clothing Type

\begin{tabular}{|l|l|c|}
\hline \multicolumn{1}{|c|}{ Clothing Type } & Humanism & Professionalism \\
\hline Business casual & $27.33(.51)$ & $39.94(.84)$ \\
\hline Business casual with lab coat & $25.27(.59)$ & $39.03(.96)$ \\
\hline Professional & $26.34(.53)$ & $40.69(.86)$ \\
\hline Professional with lab coat & $24.26(.54)$ & $38.46(.89)$ \\
\hline
\end{tabular}

lower for professionalism than all other targets.

The profile plots of estimated marginal means were examined to aid in understanding the significant interaction term (outfit type by target). The Caucasian female was rated highest on humanism when wearing professional attire with a lab coat, American female, African American male, and Caucasian male all were scored lowest on humanism when pictured in that outfit. Similarly, the professionalism rating of the Caucasian female was highest when pictured wearing the professional attire with a lab coat. The Indian female and African American female received their lowest professionalism scores in this outfit.

Participants were asked to rate their comfort having each target as their physician across health care settings. A MANCOVA indicated no significant difference by clothing type for a hospital $(F[3,495]=.97$; $P>.05$ ), emergency department ( $F[3$, $493]=1.58 ; P>.05)$, or clinic ( $F[3$, $494]=.47 ; P>.05)$. There was no difference by clothing type for discussing whereas the Indian female, African

\section{Additional Analyses for Comfort and Perceived Role}


physical $(F[3,497]=.06 ; P>.05)$, emotional $(F[3,496]=.16 ; P>.05)$, or sexual health $(F[3,495]=.44 ; P>.05)$.

Participants were asked to classify the target as a doctor, nurse, medical assistant, medical transporter, receptionist, lab technician, patient, or other. There was a lack of agreement as to what each outfit type might signify. For example, targets wearing casual attire with a lab coat were identified as a laboratory technician by 42 participants, a physician by 33 participants, and a medical assistant by 22 participants. Some participants rated the target as a physician regardless of outfit type, but professional attire with a lab coat was most associated with the physician role. Professional attire without the lab coat was most associated with a receptionist role. Female target individuals were most likely to be identified as receptionists or patients regardless of clothing type, whereas male target individuals were most likely to be identified as physicians or laboratory technicians.

\section{Preferred Clothing}

Participants were asked to rate or choose a variety of clothing, other items (eg, name tags) or other appearance factors. For clothing, medical (scrubs with or without lab coat), professional (dress slacks/dress shirt, skirt/blouse), formal (suit, dress), business casual (khaki pants/polo shirt), or casual wear (jeans, Capri pants, T-shirts) were rated. Participants were equally likely (approximately $30 \%$ ) to choose professional and medical clothing for male and female participants in an outpatient clinic. Ratings for Caucasian and minority participant ratings were similar. Younger participants and those not working in the medical setting were much more likely to prefer physicians (male and female) wear medical clothing (50\%-60\%), while older participants and those working in health care were more likely to prefer physicians (male and female) wear professional clothing (45\%$55 \%)$. Regardless of age, physicians preferred their male and female colleagues wear professional clothing (50\%-55\%)

When considering other clothing items, participants, across all subgroups, rated the following items as unacceptable (mean $<4$ on 7 -point Likert scale): jeans, T-shirts, capri pants, shorts, flip flops, sandals, sneakers, Crocs, and boots. Additionally, subgroup analysis indicated certain items were unacceptable for female physicians. Younger participants indicated a dress was unacceptable, while older participants included high heels and a skirt with no panty hose as unacceptable. Minority participants included no panty hose (dress or skirt) as unacceptable.

Other aspects of physician appearance and attire were also rated for importance (7-point Likert scale). The only item to achieve a mean score over four or acceptable was a name tag $(M=4.60)$. Subgroups were analyzed using independent $t$-tests. A name tag was significantly more important for younger participants than older participants $(t$ [474] $=2.14$; $P=.03$ ), as well as for those working in a health care setting compared to those who do not $(t[490]=-2.06$; $P=.04)$. Minority participants rated a name tag $(t[500]=-3.42 ; P=.01)$, a white coat $(t[503]=-3.08 ; P=.02)$, and a tie (if male $t[501]=-3.02, P=.03$ ) as more important than Caucasian participants. A lab coat was more important for those working in the medical field than those who do not work in medicine $(t[493]=-2.45 ; P=.02)$. Participants not in health care were less bothered by physicians with visible tattoos $(t[490]=2.04 ; P=.04)$.

\section{Discussion}

Casual attire has become more commonplace, and this has filtered into the physician's office, with more medical office staff wearing scrubs rather than traditional work attire..$^{14}$ Reflecting this fashion trend, results for the current study were somewhat inconsistent with previous studies that found patients prefer physicians wear a lab coat with professional or business clothing. ${ }^{7-10}$ While controlling for perceived attractiveness, the current study found that physicians were perceived as warmer and kinder when not wearing a lab coat. Further, a lab coat did not increase perceptions of professionalism for physicians. Prior studies have indicated that professional attire was particularly associated with greater perceptions of trust and confidence in physicians. ${ }^{8}$ However, the participants in our study indicated comfort discussing sensitive issues with physicians across medical settings, regardless of clothing type.

Previous studies of physician attire concluded that professional attire with a white coat should be the physician uniform..$^{11,15}$ However, a white coat did not guarantee the participants (medical professionals and others) identified the target as a physician. Only when paired with professional attire was it most often associated with the physician role, particularly for males. Younger participants and those who did not work in medical settings preferred physicians to wear medical clothing such as scrubs. However, wearing a lab coat and tie was more important to minority participants. Consistent with prior research, ${ }^{11}$ the participants agreed that physician attire should never be too casual.

Gjerdingen et al asserted that a name tag clearly defines a physician and such definitional symbols are important. ${ }^{11,15}$ For the current sample, wearing a name tag was the only item rated as very important by all participants. This finding was particularly pronounced for younger and minority participants and for those working in the medical field. It has been suggested that patients may be frustrated and confused by the number of medical staff encountered during a typical appointment. ${ }^{14}$ Thus, a name tag may be even more vital to identifying medical role.

It was hypothesized that non-Caucasian and female physicians would be judged more negatively when dressed less formally. This hypothesis was partially supported. For males, there were no differences on 
humanism between Caucasian and non-Caucasian targets. Contrary to our hypotheses, the African American male target was rated higher on professionalism than the Caucasian male. For female physicians, the impact of attire may be even more important, but the findings were not as straightforward as the hypothesis. After controlling for perceived attractiveness, only the Caucasian female was generally rated more favorably, particularly when wearing a lab coat. While the Caucasian female target was rated highest on both humanism and professionalism when wearing professional attire with a lab coat, the Indian and African American female targets were perceived most negatively in that outfit. Regardless of outfit type, female targets were more likely to be identified as patients or receptionists, which is consistent with prior research. ${ }^{12}$ Participants also expressed strong opinions about the appropriateness of some female clothing for physicians. Younger participants did not rate dresses favorably, while older and minority participants found bare legs and high heels unacceptable. Thus, the clearest conclusion is that attire seems to present greater and more confusing consequences for female physicians.

It was hypothesized that older physicians and patients would have greater expectations of formality in dress. Results were mixed for this hypothesis. Business casual was rated highly for humanism (warmth, kindness, etc) by older participants and all physicians, particularly older ones. The importance of more formal attire seemed to be most pronounced for professionalism as older physicians rated professional attire higher than dressing more casually, even with a lab coat. Physicians, regardless of age, showed a preference for colleagues to wear professional attire and all health care professionals were less tolerant of tattoos. Similarly, older participants preferred professional clothing for physicians, whereas younger participants preferred medical clothing (eg, scrubs).

Although compelling, there are drawbacks to this study. The results could be attributed to idiosyncrasies regarding the photographs or models. Different preferences for physician appearance have been demonstrated in other countries, therefore the results should only be applied to US settings. ${ }^{16-18}$ Further, although significant, the magnitude of the differences for humanism and professionalism should also be considered when interpreting the results. Strengths of the study include controlling for attractiveness and familiarity, which was not done in prior studies on physician attire. Taken together, the results of the study indicate three primary findings that should inform the teaching of professionalism and serve as a caution for physicians, particularly females, about the impact of attire. First, name tags are crucial to assist patients in navigating health care settings. Second, attire should never be too casual. Third, contrary to prior findings,,${ }^{11,15}$ wearing a white lab coat was not considered a particularly important component of the physician uniform. In fact, it may be detrimental for some physicians.

CORRESPONDING AUTHOR: Address correspondence to Dr Paula J. Varnado-Sullivan, Department of Psychology, Southeastern Louisiana University, SLU Box 10831, Hammond, LA 70402. pvarnado@selu.edu.

\section{References}

1. Dion K, Berscheid E, Walster E. What is beautiful is good. J Pers Soc Psychol. 1972;24(3):285290.

2. Griffin AM, Langlois JH. Stereotype directionality and attractiveness stereotyping: is beauty good or is ugly bad? Soc Cogn. 2006;24(2):187206.

3. Kaiser S. The Social Psychology of Clothing. New York: Macmillan Publishing Company; 1990.
4. Brandt LJ. On the value of an old dress code in the new millennium. Arch Intern Med. 2003;163(11):1277-1281.

5. Dunn JJ, Lee TH, Percelay JM, Fitz JG, Goldman L. Patient and house officer attitudes on physician attire and etiquette. JAMA. 1987;257(1):65-68.

6. Friedman V. The end of the office dress code. The New York Times. May 25, 2016. http:// www.nytimes.com/2016/05/26/fashion/officefashion-uniforms.html?_r=0. Accessed April 16, 2019.

7. Petrilli CM, Mack M, Petrilli JJ, Hickner A, Saint S, Chopra V. Understanding the role of physician attire on patient perceptions: a systematic review of the literature - targeting attire to improve likelihood of rapport (TAILOR) investigators. BMJ Open. 2015;5(1):e006578.

8. Rehman SU, Nietert PJ, Cope DW, Kilpatrick AO. What to wear today? Effect of doctor's attire on the trust and confidence of patients. Am J Med. 2005;118(11):1279-1286.

9. Keenum AJ, Wallace LS, Stevens AR. Patients' attitudes regarding physical characteristics of family practice physicians. South Med J. 2003;96(12):1190-1194.

10. Lill MM, Wilkinson TJ. Judging a book by its cover: descriptive survey of patients' preferences for doctors' appearance and mode of address. BMJ. 2005;331(7531):1524-1527.

11. Gjerdingen DK, Simpson DE. Physicians' attitudes about their professional appearance. Fam Pract Res J. 1989;9(1):57-64.

12. McNaughton-Filion L, Chen JS, Norton PG. The physician's appearance. Fam Med. 1991;23(3):208-211.

13. Newman AW, Wright SW, Wrenn KD, Bernard A. Should physicians have facial piercings? J Gen Intern Med. 2005;20(3):213-218.

14. Aukerman DF. Patient perception of physician appearance: a concern for all physicians. South Med J. 2003;96(12):1172-1173.

15. Gjerdingen DK, Simpson DE, Titus SL. Patients' and physicians' attitudes regarding the physician's professional appearance. Arch Intern Med. 1987;147(7):1209-1212.

16. Ikusaka M, Kamegai M, Sunaga T, et al. Patients' attitude toward consultations by a physician without a white coat in Japan. Intern Med. 1999;38(7):533-536.

17. Sotgiu G, Nieddu P, Mameli L, et al. Evidence for preferences of Italian patients for physician attire. Patient Prefer Adherence. 2012;6:361367.

18. Kurihara H, Maeno T, Maeno T. Importance of physicians' attire: factors influencing the impression it makes on patients, a cross-sectional study. Asia Pac Fam Med. 2014;13(1):2. 